\title{
Evaluación de una atención integrada contra la desnutrición infantil en áreas urbanas de Nayarit, México
}

\author{
Fernando López-Flores, MC ,MSP, ${ }_{1}^{(1)}$ Simón Barquera-C ervera, MC , M en C, ${ }^{(2)}$ \\ Ma de la Luz Kageyama-Escobar, MC, MSP.(2)
}

\section{López-Flores F, Barquera-Cervera S, Kageyama-Escobar ML. Evaluación de una atención integrada contra la desnutrición infantil en áreas urbanas de Nayarit, México. Salud Publica Mex 2004;46:158-163. El texto completo en inglés de este artículo está} disponible en: http://www.insp.mx/salud/index.html

\section{Resumen}

Objetivo. D eterminar la cobertura de laA tención Integrada a las Enfermedades Prevalentes de la Infancia y estimar la prevalencia de desmedro en niños de 6 a 36 meses de edad en dos localidades urbanas de $\mathrm{N}$ ayarit, México, para analizar su asociación. Material y métodos Estudio transversal, descriptivo y analítico, realizado en agosto de 2002 en dos áreas urbanas de $N$ ayarit, México. Se aplicó en el domicilio un cuestionario estructurado para recabar datos socioeconómicos y sobre antropometría, atención a la salud del niño y la madre, y morbilidad. Se compararon grupos con pruebas de ji2 o exacta de Fisher. Las asociaciones fueron analizadas utilizando modelos de regresión logística. Resultados La cobertura de la Atención Integrada a las Enfermedades Prevalentes de la Infancia fue de $97 \%$ en vacunación, $66.9 \%$ en suplementación con vitamina A, $91.5 \%$ en desparasitación intestinal con albendazol, $50 \%$ en vigilancia nutricia, $25.5 \%$ en capacitación materna, $24.4 \%$ en atención a la salud de la madre y $2.5 \%$ global. Se encontró una prevalencia de desmedro de $4.9 \%$, la cual estuvo asociada significativamente a esquemas incompletos, tanto de vitamina A como de albendazol. Conclusiones Los componentes de la Atención Integrada a las Enfermedades Prevalentes de la Infancia muestran coberturas acordes con su factibilidad y tiempo de implantación. Los hallazgos de la asociación de la atención integrada de la salud infantil con el desmedro destacan la importancia de reunir mayor evidencia sobre su

\author{
López-Flores F, Barquera-Cervera S, Kageyama-Escobar ML. \\ Evaluation of integrated management \\ of childhood illness against malnutrition \\ in urban areas of Nayarit, Mexico. \\ Salud Publica Mex 2004;46:158-163. \\ The English version of this paper \\ is available at: http://www.insp.mx/salud/index.html
}

\section{A bstract \\ Abstract}

O bjective. To assess the association between coverage of the Integrated Management of Childhood IIIness (IMCI) strategy and the prevalence of stunting in children 6 to 36 months of age. Material and Methods A cross-sectional study was conducted in two urban areas of $\mathrm{N}$ ayarit, Mexico, in A ugust 2002. Anthropometry, health care for mothers and their children, morbidity and socioeconomic data were collected by means of household interviews. Statistical analyses were performed with chi-squared tests, Fisher's exact test, and logistic regression. Results $\mathrm{IMCl}$ coverage rates were as follows: immunization $97 \%$, vitamin A supplementation $66.9 \%$, dew orming with albendazole $91.5 \%$, nutritional surveillance $50 \%$, maternal health education $25.5 \%$, maternal health care $24.4 \%$, and $2.5 \%$ overall. The prevalence of stunting was $4.9 \%$, and was statistically associated with incomplete courses of vitamin A supplementation and albendazo le deworming. Conclusions. Coverage of $\mathrm{IMCI}$ components varied according to their feasibility and time of implementation. The associations between IMCI and failure to thrive highlight the importance of obtaining more information about its effects on the nutritional status of children. This will help to improve prevention strategies against child malnutrition. The English version of this paper is available at:http://www.insp.mx/salud/ index.html

(1) Secretaría de Salud de N ayarit. Tepic, N ayarit, México.

(2) Instituto $N$ acional de Salud Pública. Cuernavaca, Morelos, México.

Fecha de recibido: 5 de agosto de 2003 - Fecha de aprobado: 9 de marzo de 2004 Solicitud de sobretiros: Dr. Fernando López Flores. Secretaría de Salud de $N$ ayarit. D epartamento de Medicina Preventiva. Calle Dr. Gustavo Baz N o. 33 Sur, Fraccionamiento Fray Junípero Serra 63190.Tepic, N ayarit. Correo electrónico: jferlf@ hotmail.com 
efecto en el estado nutricio, con el fin de contribuir en el mejoramiento y prevención de la desnutrición infantil. El texto completo en inglés de este artículo está disponible en: http://www.insp.mx/salud/index.html

Palabras clave: atención integral de salud; desnutrición proteico-ener gética; cobertura de servicios de salud; M éxico
Key words: comprehensive health care; protein-energy malnutrition; health services coverage; M exico
$\mathrm{L}$ a Atención Integrada a las Enfermedades Prevalentes de la Infancia (AIEPI) fue elaborada por la Organización Mundial de la Salud (OMS) y el Fondo de las Naciones Unidas para la Infancia (UNICEF), y presentada en 1996 como la principal estrategia para mejorar la salud de la niñez. La AIEPI combina la adecuada atención institucional y comunitaria de las enfermedades que prevalecen en la infancia con factores que influyen en la salud infantil como la nutrición, la vacunación y la salud materna. ${ }^{1,2}$

En México, a lo largo de varias décadas, se han implementado diversos programas y políticas orientados a mejorar la nutrición de grupos vulnerables, sin embargo, es hasta principios de los años 90 cuando se iniciaron programas integrados, que abarcan componentes de salud, educación, distribución de alimentos y mejoras en los servicios públicos de las comunidades. ${ }^{3}$ En 1998 la Secretaría de Salud adopta la AIEPI con el propósito de mejorar los niveles de salud de los niños menores de cinco años de edad a través de acciones que incluyen vigilancia de la vacunación y de la nutrición, atención del motivo de la consulta, así como capacitación y atención a la salud de la madre. ${ }^{4}$

En Nayarit, la AIEPI dio inicio en mayo de 1998 en población de responsabilidad de la Secretaría de Salud de Nayarit (SSN) de la localidad urbana de Acaponeta, extendiéndose paulatinamente al resto de las unidades médicas de la SSN, entre ellas la de Santiago Ixcuintla.

Las causas de la desnutrición son multisectoriales y abarcan la alimentación, la salud, las prácticas de atención, el saneamiento, los servicios de salud y factores sociales. ${ }^{5}$ En México, según datos de la Encuesta Nacional de Nutrición 1999 (ENN-99), la prevalencia de baja talla para la edad o desmedro en menores de cinco años fue de $17.8 \%$. En la región centro, a la que pertenece el estado de Nayarit, la prevalencia de desmedro en este grupo de edad, fue de $14.5 \%$, y en área urbana de $10.4 \% .^{6}$

Diversos estudios alrededor del mundo, incluyendo a México, han evaluado las actividades y el impacto de la AIEPI en la salud infantil, sin embargo, la asocia- ción que ésta tiene con el estado nutricio de los niños, ha sido poco explorada. $1,7-10$

Los objetivos del presente estudio fueron: a) determinar la cobertura de la AIEPI en población asignada a la SSN de las localidades urbanas de Acaponeta y Santiago Ixcuintla; b) estimar la prevalencia de desmedro en niños de 6 a 36 meses de edad en las áreas de estudio; c) comparar las prevalencias de desmedro encontradas entre Acaponeta y Santiago Ixcuintla; d) comparar las prevalencias de desmedro de las localidades de estudio con la de la ENN-99, y e) evaluar la asociación que existe entre los componentes de la AIEPI y el desmedro de la población estudiada.

\section{Material y métodos}

Se realizó un estudio transversal durante el mes de agosto de 2002, en población infantil de 6 a 36 meses de edad de las localidades urbanas de Acaponeta y Santiago Ixcuintla, ubicadas en la Jurisdicción Sanitaria III del estado de Nayarit, México.

Los tamaños de las muestras fueron de 181 niños para cada localidad, calculadas con un nivel de confianza de $95 \%$, un poder estadístico de $80 \%$, una prevalencia teórica de desmedro de $4 \%$ en Acaponeta y de $8 \%$ en Santiago Ixcuintla, y seleccionadas aleatoriamente. Se excluyeron aquellos niños que presentaron bajo peso al nacer (menor a $2500 \mathrm{~g}$ ), malformación congénita o presencia de enfermedad crónica.

Previa prueba piloto y con el consentimiento informado de la madre o responsable del menor, a cada participante se le aplicó en su domicilio un cuestionario estructurado para recoger información sobre datos de identificación, de atención a la salud del niño y de la madre, de antropometría y de características socioeconómicas.

La toma de mediciones antropométricas se llevó a cabo con las técnicas descritas y recomendadas por la OMS, ${ }^{11}$ y con personal estandarizado con la metodología propuesta por Habicht. ${ }^{12}$

Se obtuvo la talla de los niños de 6 a 23 meses de edad mediante infantómetros de acrílico Dimedic ${ }^{\circledR}$ con 
precisión de $0.5 \mathrm{~cm}$, y de los niños de 24 a 36 meses de edad con estadímetros de pared Dimedic ${ }^{\circledR}$ con precisión de $1 \mathrm{~mm}$. El peso se midió con básculas de resorte tipo Salter Dynatop ${ }^{\circledR}$, con capacidad de $20 \mathrm{~kg}$ y precisión de $100 \mathrm{~g}$.

La variable dependiente fue desmedro, definida como talla para edad menor a -2 desviaciones estándar del patrón de referencia de la OMS / National Center for Health Statistics (OMS/ NCHS); ${ }^{11}$ y como variables independientes se consideraron: 1) el esquema completo de vacunación, que en el grupo de edad de 6 a 11 meses consistió en una dosis de vacuna antituberculosa (BCG), tres dosis de vacuna antipoliomielítica (Sabin) y tres dosis de vacuna pentavalente; y en los niños de 12 a 36 meses de edad, además del esquema anterior, una dosis de vacuna contra sarampión, rubéola y parotiditis (SRP); 2) vigilancia de la nutrición: toma de peso y talla al menos a los 2, 4, 6, 12 y 24 meses acorde con su edad; 3 ) suplementación con vitamina A: administración oral de 100000 UI de vitamina A en niños de 6 a 11 meses de edad y de 200000 UI en el grupo de edad 12 a 36 meses, durante las dos Semanas Nacionales de Salud previas (octubre de 2001 y mayo de 2002); 4) desparasitación intestinal: administración de $400 \mathrm{mg}$ de albendazol a niños mayores de dos años de edad durante las Semanas Nacionales de Salud mencionadas; 5) capacitación de la madre: se clasificó como madre capacitada aquella que refirió haber tenido en los últimos 12 meses tres o más sesiones de capacitación sobre la atención a la salud del niño, y 6) atención a la salud de la madre: madres que tuvieron las cuatro atenciones médicas siguientes: una o más tomas de Papanicolaou en los últimos tres años, una o más tomas de presión arterial y de peso en el último año, y dos o más dosis de vacuna antitetánica.

Se realizó análisis descriptivo empleando medias y desviaciones estándar para datos continuos, y proporciones para variables categóricas. Se efectuó análisis comparativo con la pruebas de Ji cuadrada, exacta de Fisher y $t$ de Student. Para identificar variables asociadas a desmedro se utilizó análisis multivariado por regresión logística, observando razones de momios (RM), intervalos de confianza de 95\% (IC 95\%) y valores $p$; se incluyeron aquellas variables que en el análisis bivariado tuvieron un valor de $p<0.20$, y quedaron en el modelo final las que presentaron valores de $p<0.05$, ajustándose por edad, sexo, morbilidad y nivel socioeconómico. Se evaluaron interacciones, así como el ajuste del modelo con la prueba de Hosmer-Lemeshow. El nivel aceptable de significancia estadística para todas las pruebas fue de $p<0.05$. Los datos se analizaron con el paquete estadístico Stata 7.0.

\section{Resultados}

En el estudio participaron 365 niños (46\% varones y $54 \%$ mujeres), de los cuales 182 fueron de Acaponeta y 183 de Santiago Ixcuintla.

La cobertura de la AIEPI fue de $97 \%$ en esquema completo de vacunación, $66.9 \%$ en suplementación con vitamina A, 91.5\% en desparasitación intestinal con albendazol, $50 \%$ en vigilancia nutricia, $25.5 \%$ en capacitación materna, $24.4 \%$ en atención a la salud de la madre y $2.5 \%$ global. La figura 1 muestra las coberturas de los componentes de la AIEPI por localidad, las cuales fueron significativamente más bajas en Santiago Ixcuintla respecto a Acaponeta, la de esquema completo de vacunación $(p=0.02)$ y la de vigilancia nutricia $(p=0.03)$. En Acaponeta 3.3\% de los niños presentó todas las intervenciones de la AIEPI; mientras que en Santiago Ixcuintla sólo 1.6\% ( $p=0.31)$.

En el cuadro I se presenta la prevalencia de desmedro por localidad y grupo de edad, siendo mínima en los niños más pequeños y aumentando conforme el grupo de edad era mayor $(p=0.14)$. En Acaponeta la prevalencia de desmedro fue $35.9 \%$ menor que en Santiago Ixcuintla, sin embargo, esta diferencia no resultó estadísticamente significativa $(p=0.34)$, ni aun por grupos de edad. La prevalencia de desmedro encontrada en Acaponeta fue $62.5 \%$ menor a la reportada en la ENN99 para menores de cinco años de edad de área urbana en la región centro (3.9\% vs. $10.4 \%, p=0.002)$; en San-

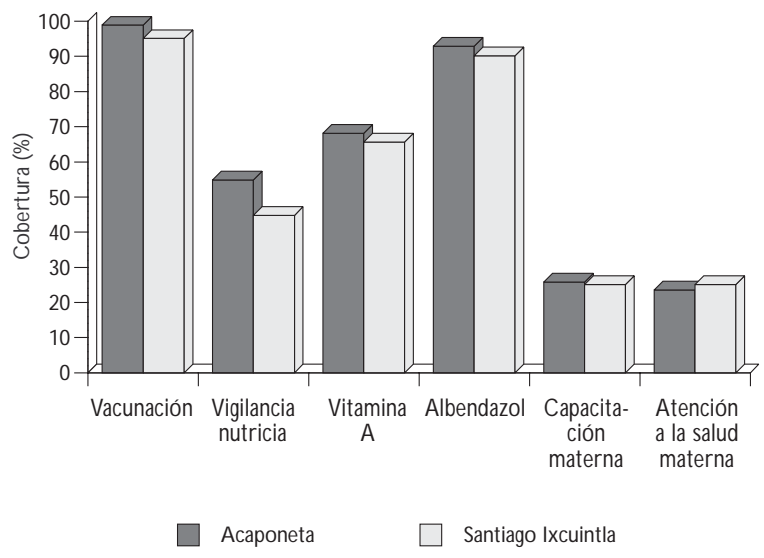

Figura 1. Cobertura de los componentes de la Atención Integrada a las Enfermedades Prevalentes de la INFANCIA POR LOCALIDAd. NAYARIT,MéXICO, 2002 


\section{Cuadro I \\ Prevalencia de desmedro por localidad Y GRUPO de EDAD. Nayarit, México, 2002}

\begin{tabular}{|c|c|c|c|c|c|c|c|c|c|}
\hline \multirow{2}{*}{$\begin{array}{l}\text { Grupos de edad } \\
\text { en meses }\end{array}$} & \multicolumn{3}{|c|}{ Acaponeta } & \multicolumn{3}{|c|}{ Santiago Ixcuintla } & \multicolumn{3}{|c|}{ Global } \\
\hline & $n$ & CasOs & $\%$ & $\mathrm{n}$ & casos & $\%$ & $n$ & casos & $\%$ \\
\hline $6-11$ & 39 & 0 & 0.0 & 45 & 1 & 2.2 & 84 & 1 & 1.2 \\
\hline $12-23$ & 66 & 3 & 4.6 & 74 & 4 & 5.4 & 140 & 7 & 5.0 \\
\hline $24-36$ & 77 & 4 & 5.2 & 64 & 6 & 9.4 & 141 & 10 & 7.1 \\
\hline Total & 182 & 7 & 3.9 & 183 & 11 & 6.0 & 365 & 18 & 4.9 \\
\hline
\end{tabular}

tiago Ixcuintla esta diferencia fue de $42.3 \%$ ( $6 \%$ vs. $10.4 \%, p=0.03)$, y global de $52.9 \%$ ( $4.9 \%$ vs. $10.4 \%, p<$ 0.001). Asimismo, en todos los niños se observó menor prevalencia de desmedro a mayor número de intervenciones de la AIEPI $(p=0.02)$.

En el modelo de regresión logística ajustado por edad, sexo y enfermedad, resultaron como factores asociados a desmedro el esquema incompleto de vitamina A(RM 5.0, IC 95\% 1.2-21.1, $p=0.03$ ), el esquema incompleto de albendazol (RM 8.0, IC 95\% 1.1-58.6, $p=0.04$ ), y el nivel socioeconómico bajo (RM 14.9, IC $95 \%$ 3.6-60.7, $p<0.001$ ). El peso para edad, representado en unidades de desviación estándar, resultó un factor protector para desmedro (RM 0.075, IC 95\% $0.02-0.25, p<0.001)$.

\section{Discusión}

La evaluación de la AIEPI en el ámbito comunitario nos revela coberturas por componente variadas y en conjunto bajas, similares en ambas localidades del estudio y acordes con su tiempo de desarrollo, prioridad, asignación de recursos y dificultad para llevarse a cabo. La vacunación infantil, con más de cinco décadas de evolución y de alta prioridad por razones epidemiológicas, ${ }^{13}$ presenta coberturas mayores a $95 \%$, comparables con los reportes estatales y nacionales, que aunque en el presente estudio no se asociaron con desmedro, se conoce el impacto negativo que pueden tener algunas enfermedades inmunoprevenibles como lo es el sarampión en la nutrición. ${ }^{14-16}$

La Organización Panamericana de la Salud reporta coberturas de suplementación con vitamina A mayores a $60 \%$ en niños de 6 a 11 meses de edad y de 34 a $75 \%$ en mayores de un año de edad de países latinoamericanos, ${ }^{17}$ congruentes con 87 y $61 \%$, respectivamente, encontrado en este estudio. Estos hallazgos posiblemen- te se deban al frecuente contacto que este grupo de edad tiene con los servicios de salud, en especial con el de inmunizaciones, así como a las campañas nacionales de salud y al enfoque de atención integrada. En México se ha demostrado que la suplementación con vitamina A mejora los niveles de retinol por periodos no mayores a tres meses, aunque elimina las deficiencias severas de la vitamina. ${ }^{18}$ Varios estudios de tipo transversal han encontrado asociación entre deficiencia de vitamina A y desmedro, sin embargo, en ensayos aleatorizados no se ha encontrado mejoría en ganancia de talla, aunque sí mejora el peso y reduce la severidad de la diarrea. $15,16,19,20$

El tratamiento periódico masivo con albendazol resulta un medio de control más efectivo contra la parasitosis intestinal que utilizado de manera selectiva, y tiene mayor efecto sobre la intensidad de la reinfección que sobre su prevalencia. ${ }^{21-23}$ La disentería ocasionada por el Trichuris trichiura está asociada con desmedro, con anemia por deficiencia de hierro y con efectos adversos en la función cognoscitiva; mientras que el $A s-$ caris lumbricoides puede causar mala absorción de vitamina A, por lo que se recomienda que las intervenciones de suplementación nutricia se apoyen con tratamiento antihelmíntico, el cual resulta efectivo, seguro y barato. ${ }^{24-26}$

El monitoreo del crecimiento infantil se ha evaluado en India como parte de una serie de intervenciones integradas, sin resultado benéfico sobre la talla, el cual resulta ser efectivo si el estatus de la mujer es mejor, si las familias tienen recursos suficientes para atender a sus niños, si existe cohesión comunitaria y si la infraestructura de la atención primaria a la salud está desarrollada. ${ }^{27} \mathrm{El}$ registro del crecimiento ayuda a documentar el progreso y estado de salud de los niños, e indirectamente la efectividad de los sistemas de salud y, en conjunto con la educación, parece tener un impacto positivo en el comportamiento materno relacionado con la nutrición del niño. ${ }^{28}$

En contraste con $25 \%$ de madres capacitadas encontrado en este estudio, en una evaluación del impacto de la AIEPI en México, se determinó que 58\% de las madres tenía una adecuada capacitación en atención del niño enfermo en el hogar. ${ }^{10}$ Esto sugiere la necesidad de crear procesos educativos que promuevan la comprensión de su situación de salud, así como el desarrollo de actitudes, habilidades y destrezas, ${ }^{29}$ y que además sean culturalmente aceptados por la comunidad, de probada efectividad, económicamente viables ${ }^{30} \mathrm{y}$ enfocados a los comportamientos necesarios de ser cambiados en la población blanco, ${ }^{31}$ 
destacándose la necesidad de una participación consciente y previo conocimiento de hábitos, costumbres y valores de la población. ${ }^{32}$

Algunos autores consideran importante evaluar el estado de salud de la madre, ya que es difícil encontrar un niño saludable con una madre enferma, quien si desconoce la importancia de realizarse evaluaciones médicas periódicas, es posible que ignore las necesidades de salud del niño. ${ }^{10}$

La tendencia ascendente de prevalencia de desmedro, conforme la edad es mayor, encontrada en este estudio es consistente con la del ámbito mundial y semejante a la observada en México. ${ }^{6,33}$ Las diferentes prevalencias de desmedro entre las áreas de estudio y la ENN-99 sugieren considerar un esfuerzo conjunto para obtener información de calidad sobre nutrición cada vez más focalizada que apoye las políticas de salud y la toma de decisiones en los diferentes niveles de atención.

Bobadilla y colaboradores documentaron que para prestar servicios de salud que permitan atender las necesidades de una población conviene considerar como criterios básicos el peso de la carga ocasionada por un factor de riesgo y la efectividad de las intervenciones para evitarlo en función del costo. Esto justifica agrupar intervenciones que minimicen su costo total, que optimicen los recursos y que reduzcan el costo de obtención de servicios realizados por la población. ${ }^{34}$ Por su parte, la OMS considera que los programas "verticales" obtienen resultados tangibles pero no duraderos; por el contrario, los programas integrados son sostenibles, eficientes y cómodos para los usuarios, esto se ha demostrado por décadas en países en desarrollo. ${ }^{35}$

Resulta conveniente monitorear y evaluar este programa de atención integrada de la salud infantil a mayor escala y mediante diseños epidemiológicos que logren reunir mayor evidencia sobre su efecto sinérgico en el estado nutricio para aprovecharlo con eficiencia, $\mathrm{y}$ valorar nuevas intervenciones viables hasta que los beneficios adicionales no justifiquen nuevos incrementos del $\operatorname{costo}^{36} \mathrm{y}$, sobre todo, que sea focalizado hacia quienes más lo necesitan y en donde el número de beneficiarios sea mayor, con el fin de acelerar las modestas tendencias descendentes de desnutrición infantil observadas en las últimas tres décadas en México. ${ }^{30,37}$

\section{A gradecimientos}

Agradecemos la valiosa participación del doctor Mario Flores Aldana en la elaboración de este documento, así como también a la Secretaría de Salud de Nayarit, a las autoridades y personal de vacunación de la Jurisdicción Sanitaria III de Tuxpan, y a las Coordinaciones
Médicas Municipales de Acaponeta y Santiago Ixcuintla por su colaboración y facilidades brindadas en la realización del trabajo de campo.

\section{Referencias}

1. O rganización Panamericana de la Salud/O rganización Mundial de la Salud. Implementación de la estrategiaA IEPI en las A méricas: avances y desafíos. W ashington, D C: O PS/O MS; 2002. (SPP36/5).

2.0 rganización Mundial de la Salud. División de Salud y D esarrollo del $N$ iño.A IEPI información.A tención a las enfermedades prevalentes de la infancia: justificación de una estrategia integrada. G inebra: O MS; 1997. 3. Barquera S, Rivera-D ommarco J,G asca-G arcíaA . Políticas y programas de alimentación y nutrición en México. Salud Publica Mex 2001;43: 464-477.

4. Secretaría de Salud. N orma 0 ficial Mexicana N O M-031-SSA2-1999, para la Atención a la Salud del N iño. México, D F: D iario 0 ficial de la Federación; 2001.

5. Fondo de las $\mathrm{N}$ aciones Unidas para la Infancia. Estado mundial de la infancia. Tema: nutrición. N ueva York (N Y): UN IC EF; 1998.

6. Rivera-D ommarco J, Shamah-LevyT,Villalpando-Hernández S, G onzález de Cossío T, Hernández-Prado B, SepúlvedaJ. Encuesta $N$ acional de N utrición 1999. Estado nutricio de niños y mujeres en México.

Cuernavaca, Morelos, México: Instituto N acional de Salud Pública; 2001. 7. Lambrechts T,Bryce J, 0 rindaV.A tención integrada a las enfermedades prevalentes de la infancia: resumen de las primeras experiencias. Bull W orld Health 0 rgan 1999;77:582-594.

8. Bern C, Zucker J, Perkins B, 0 tieno J, 0 loo A,Yip R.Assessment of potential indicators for protein-energy malnutrition in the algo rithm for integrated management of childhood illness. Bull W orld H ealth 0 rgan 1997;75 Suppl 1:87-96.

9. Somoes E, D estaT,TessemaT, G erbresellassie T, D agnew M, G ove S. Performance of health workers after training in integrated management of childhood illness in Gondar, Ethio pia. BullW orld Health 0 rgan 1997;75 Suppl 1:43-53.

10. G uiscafré H, Martínez H, Palafox M ,Villa S, Espinosa P, Bojalil R et al. The impact of a clinical training unit on integrated child health care in Mexico. BullW orld Health 0 rgan 2001;79:434-441.

11. 0 rganización Mundial de la Salud. Medición del cambio del estado nutricional. Ginebra: O MS; 1993.

12. Habicht J. Estandarización de métodos epidemioló gicos cuantitativos sobre el terreno. Bol 0 ficina Sanit Panam 1974;76:375-385.

13. Secretaría de Salud. Consejo N acional deVacunación. Memoria 19901994. México, DF: SSA; 1994.

14. Rivera J, Long K, G onzálezT, Parra S, Rivera M, Rosado J. C uadernos de salud. N utrición y salud: un menú para la familia. México, DF: SSA; 1994.

15. Hussey G, Klein M.A randomized, controlled trial of vitaminA in children with severe measles. N Engl J Med 1990;323:160-164.

16. Coutsoudis A, Broughton M, C oovadia H.Vitamin A supplementation reduces measles morbidity in young $A$ frican children:A randomized, placebo-controlled, double blind trial.A m J C lin N utr 1991;54:890-895. 17. 0 rganización Panamericana de la Salud/ 0 rganización Mundial de la Salud.Visión integrada de la suplementación con vitaminaA en las A méricas. Washington, DC: O PS/O MS; 2001. (HPP/HPN/MN/49-17). 18. Robles-Sardin AE,A stiazarán-G arcía H, D ávalos-N avarro R, Q uihuiCota L, C abrera-Pacheco RM,Valencia ME.Efecto de la suplementación con una dosis masiva de vitaminaA en niños de 6 a 36 meses de edad. Salud Publica Mex 1998;40:309-315.

19.W est K, D junaedi E, PandjiA, Kusdiono,Tarwotjo I,Sommer A.Vitamin A supplementation and growth:Randomized community trial.A $\mathrm{m} \mathrm{J} \mathrm{C} \mathrm{lin}$ N utr 1988;48:1257-1264. 
20. Ramakrishnan U, Martorell R. The role of vitamin A in reducing child mortality and morbility and improving growth. Salud Publica Mex 1998:40:189-198.

21. Hall A,Anwar K,Tomkins A. Intensity of reinfection with Ascaris lumbricoides and its implications for parasite control. Lancet 1992;339:1253-1257.

22. Beltramino D, Lurá M, C arrera E. El tratamiento antihelmíntico selectivo frente al tratamiento masivo. Experiencia en dos comunidades hiperendémicas. Rev Panam Salud Publica 2003;13:10-17.

23. Belizario V,A marillo M, D e León W, D e los Reyes A, Bugayong M, Macatangay B.A comparison of the efficacy of single doses of albendazole, ivermectin, and diethylcarbamazine alone or in combinations agains Ascaris and Trichuris spp. BullW orld Health 0 rgan 2003;81:35-41.

24. Cooper E, Bundy D, Henry F.C hronic dysentery, stunting, and whipworm infestation. Lancet 1986;2:280-281.

25. Savioli L, Bundy D,TomkinsA. Intestinal parasitic infections:A soluble public health problem.Trans RSTrop Med Hyg 1992;86:353-354.

26. Stephenson L, Latham M, Kurz K, Kinoti S, Brigham H. Treatment with a single dose of albendazole improves growth of Kenyan schoolchildren with hookworm, Trichuris trichiura, and Ascaris lumbricoides infections.Am J Trop Med Hyg 1989;41:78-87.

27. G eorge S, Latham M,A bel R, Ethirajan N , Frongillo E. Evaluation of effectiveness of good growth monitoring in so uth Indian villages. Lancet 1993;342:348-352.

28.Tulchinsky T, Ebweini S, G insberg G , A bedY, Montano D, Schoenbaum M et al. G rowth and nutrition patterns of infants associated with a nutrition education and supplementation programme in $\mathrm{G}$ aza, 1987-92. BullW orld Health 0 rgan 1994;72:869-875.

29. Regueyra G. Construyamos juntos. En: García M, Cerqueira M, ed. Participación social y educación para la salud. Memorias del I taller subregional latinoamericano. México, DF: O MS-O PS/SSA/UIPES-O RLA/ IN N SZ, 1994:473-486.

30. Rivera J. Estrategias y acciones para corregir deficiencias nutricias. Bol Med Hosp Infant Mex 2000;57:641-649.

31. Levinson F, Rogers B, Hicks K, SchaetzelT,Troy L,Young C . Monitoring and evaluation of nutrition programs in developing countries. $N$ utr Rev 1999;57:157-164

32. Martínez H, Martínez G, Contreras J, Saucedo G, Huerta L, Ramos R et al. Experiencias en participación comunitaria para promover la educación en nutrición. Salud Publica Mex 1993;35:673-681.

33. 0 rganización Mundial de la Salud. El estado físico: uso e interpretación de la antropometría. G inebra: O MS; 1995. (Serie de Informes Técnicos $\mathrm{N}$ ㅇ 854).

34. Bobadilla J, C owley P, Musgrove P, Saxenian H. D iseño, contenido y financiamiento de un paquete nacional de servicios de salud esenciales. Bol 0 ficina Sanit Panam 1995;118:127-140.

35. 0 rganización Mundial de la Salud. Integración de la atención de salud: Informe de un Grupo de Estudio de la O MS. Ginebra: O MS; 1996. (Serie de Informes Técnicos $N$ ㅇ 861)

36. Habicht J, Butz W. Medición de los efectos de proyectos de intervención nutricional en gran escala sobre la salud y la nutrición. En: Klein R, Read M, Riecken H, Brown J Jr, PradillaA, D aza C , ed. Evaluación del impacto de programas de nutrición y salud. Washington, DC: O PS/ O MS; 1982:71-91.

37.AvilaA, C hávezA,Shamah T, Madrigal H.La desnutrición infantil en el medio rural mexicano: análisis de las encuestas nacionales de alimentación. Salud Publica Mex 1993;35:658-666. 